\title{
Subject Index Vol. 21, 2006
}

\section{Cerebrovascular Diseases}

\section{ABCA1 254}

Abciximab 1 (S1)

Actiwatches 307

Acute stroke 166, 340

Angiogenesis 154

Antiplatelet drugs 372

- therapy 35 (S1), 242

Antiplatelets 17 (S1)

Antithrombotic drug therapy before stroke 401

Aorta 26

Aortic atheroma 60

ApoE 266

Apolipoprotein E 266

Apolipoproteins 323

Apoptosis 32

Arteriovenous malformation, cerebral 154

Aspirin 1 (S1), 242, 353

- resistance 7 (S1), 98

Atherosclerosis, risk factors 91

Atherothrombosis 60

Atrial fibrillation 279, 372, 401

Barthel Index 386

Bilirubin 408

Blood flow, carotid arteries 54

Borderzone infarction 145

Cardioembolic infarction 120

Cardiovascular disease(s) 26, 187, 235, 254

Carotid arteries 223

- artery 393

- atherosclerosis 112, 408

- stenosis 393

Cerebral blood flow 297

- infarction 17 (S1), 26, 60, 74, 271, 357

- ischemia $32,353,386$

- vasoreactivity 194

Cerebrovascular disease 329

- reactivity to L-arginine 180

Cheyne-Stokes respiration 166, 340

Cilostazol 1 (S1), 7 (S1)

Citicoline 380

Clopidogrel 1 (S1), 242

Collateral circulation 74

Combination therapy 7 (S1)

Computed tomography 336

Consent procedures 348

Contrast-enhanced 3-D magnetic resonance angiography 393

(S1) = Supplement 1. Supplement 2 has its own Subject Index.

Coronary artery bypass 112

- atherosclerosis 112

- heart disease 47

Cox proportional hazards analyses 187

Diazepam 120

Diffusion MRI 106

Diffusion-weighted imaging 217, 336

Dipyridamole 1 (S1), 98

Drug-eluting stents 25 (S1)

Dual antiplatelet therapy 25 (S1)

Early CT findings, stroke 367

Echocardiography 26

Elderly 229

Electrocardiography, QT prolongation 47

E-mail follow-up 363

Embolism 145, 223

Endarterectomy 393

Endothelial dysfunction 180

External and extreme capsular stroke 217

First-ever ischemic stroke 42

Flow diversion 74

Follow-up disease management 329

Gavestinel 106

Genetics 254

Gilbert syndrome 408

GP IIb/IIIa inhibitor(s) 1 (S1), 7 (S1)

Health-related quality of life 247

Hemicraniectomy 79

Hydrocephalus 159

Hypercholesterolemia 315

Hypertension 35 (S1), 315

Hypoperfusion 145

Hypothermia 79

Hypoxaemia 166

Hypoxia 340

In vitro generation of thrombin 98 Inclusion criteria, clinical trial design 386

Informal caregiver 128

Informant Questionnaire for Cognitive Decline in the Elderly 401

Intracerebral h(a)emorrhage 159, 266, 380

Intracranial artery calcification 91

- atherosclerosis 112
Intravenous Magnesium Efficacy in Stroke trial 260

Ischaemic stroke 348

- $\quad$ - risk factors 323

Ischemic edema, early 336

- stroke outcomes 260

Lacunar infarct 315

- infarction 17 (S1), 180

Leukoaraiosis 315

Lipids 323

Long-term mortality 187

Low perfusion syndrome 145

Magnetic resonance imaging 297

- _ volumetry 307

Malignant cerebral ischemia 79

Microembolic signals 242

Molecular target 7 (S1)

Motor activity 247

- hemineglect 307

MR, diffusion-weighted image 357

Multi-detector-row computed tomography 91

Muscle mass 201

- spasticity 247

- strength 201

Myocardial infarction 60

Neuronal plasticity 6

Neuroprotection 106, 120, 380

Neurovascular coupling 194

- ultrasound 86

Nitric oxide 180, 194

- - synthase 180

Nucleosomes, DNA 32

OCSP 86

Oral anticoagulation 372

- direct thrombin inhibitor 279

Outcome 229

Percutaneous coronary intervention 25 (S1)

Perfusion-diffusion mismatch 357

Peripheral artery disease 60

Phosphodiesterase inhibitor 194

Placebo-controlled clinical trial 106

Platelet aggregation 98

Pleiotropic effect 7 (S1)

Polymorphisms 254

Positron emission tomography 336 
Prestroke dementia 401

Primary intracerebral hemorrhage 18

Prostacyclin 194

Pulmonary hypertension 194

QT prolongation 47

Quality of health care 235

Rehabilitation 6, 128, 229

Risk factors 18,254

Secondary prevention $235,353,372$

Sickle cell disease 38

Sildenafil 194

Smoking 315

- status 260

Soluble intercellular adhesion molecule 1 67

Statin 35 (S1)

Steno-occlusive disease 297

Stenosis 223
Stroke 6, 18, 32, 38, 86, 201, 229, 235, 247, $254,279,315,372,386$

- assessment 271

- deterioration, infarct-inherent mechanisms 42

- epidemiology 323

- guidelines $17(\mathrm{~S} 1)$

- incidence $17(\mathrm{~S} 1)$

- outcome 120,173

- prevention $35(\mathrm{~S} 1)$

- severity 187

- unit benefit 173

-, acute 47, 336

-, functional outcome 271

Subarachnoid hemorrhage 363

Thai stroke patient 128

Thienopyridine 7 (S1)

Thromboembolism 279

Thrombolysis 348

Ticlopidine 1 (S1)
Tracheostomy 159

Transcranial Doppler 38, 194, 223

- _ ultrasound 74

Transient ischemic attack 235

- speech arrest 217

Transitory ischemic attack 329

Ultrasonography 54

Ultrasound 194

Unknown-onset stroke 367

Vascular endothelial growth factor, plasma 154

Wake-up stroke 367

Warfarin 279

Washout syndrome 145

Watershed infarction 357

White matter changes 315

Ximelagatran 279 